\title{
¿Quiénes se fueron? Aproximaciones a los motivos de abandono de un programa de apuntalamiento a las trayectorias educativas de estudiantes universitarios
}

Who dropped out? An approach to the reasons why higher education students abandoned a socio-educational program to support their educational paths

\author{
Volumen 18, Número 2 \\ Mayo-Agosto \\ pp. 1-30
}

Este número se publica el 1 de mayo de 2018

DOI: https://doi.org/10.15517/aie.v18i2.33160

\author{
Liliana Mayer \\ Leticia Cerezo
}

Revista indizada en REDALYC, $\underline{\text { SCIELO }}$

Revista distribuida en las bases de datos:

LATINDEX, DOAJ, $\underline{\text { REDIB }}$ IRESIE, $\underline{\text { CLASE}}$, DIALNET, SHERPA/ROMEO, QUALIS-CAPES, MIAR

Revista registrada en los directorios:

ULRICH'S, $\underline{\text { REDIE}}, \underline{\text { RINACE}}, \underline{\text { OEI, MAESTROTECA, PREAL, }} \underline{\text { CLACSO }}$ 


\title{
¿Quiénes se fueron? Aproximaciones a los motivos de abandono de un programa de apuntalamiento a las trayectorias educativas de estudiantes universitarios
}

\author{
Who dropped out? An approach to the reasons why higher education students abandoned a \\ socio-educational program to support their educational paths
}

\section{Liliana Mayer Leticia Cerezo}

\begin{abstract}
Resumen: La masificación universitaria, que conlleva un incremento en la cantidad de ingresantes a las universidades, plantea situaciones novedosas a ese nivel educativo en términos de composición social del estudiantado. Distintos intentos de dar respuesta a esas problemáticas han surgido, algunos de ellos, provenientes de organizaciones de la sociedad civil. En el presente artículo presentamos los resultados de parte de una evaluación de un programa educativo de apuntalamiento a la trayectoria universitaria de jóvenes provenientes de sectores vulnerables de Argentina. Específicamente, abordamos los motivos aducidos por aquellas personas "ex - participantes" para concluir su incursión en el Programa. Con este objetivo se desplegó una investigación cuya estrategia metodológica fue cualitativa y constó de la realización de 15 entrevistas en profundidad a jóvenes que dejaron de formar parte del programa evaluado. Con el fin de dar cuenta de los repertorios que explican la salida del Programa de los "ex becarios", se los dividió en dos grandes grupos: quienes salieron del Programa por sugerencia de las organizaciones de la sociedad civil que lo gestionan, y a través de las cuales se materializaba su adhesión a la estrategia; y quienes se "salieron" por iniciativa propia. La investigación da cuenta de tendencias actuales para las trayectorias universitarias, que combinan periodos de abandono con la promesa - muchas veces cumplida - de continuar los estudios, y la necesidad de compatibilizar el estudio con el trabajo.
\end{abstract}

Palabras clave: acceso a la educación, estudiante universitario, deserción escolar, política educacional

\begin{abstract}
Higher education massification process implies, by definition, an increase in enrollment rates. Furthermore, it brings new situations in terms of the social composition of the student body. In the intent of giving responses to the new problematics that these changes suppose, there have been implemented-and still aredifferent initiatives, related to higher education inclusion. Though most of them are State initiatives, in a smaller amount, others came from NGOs. In the following article, we present part of the results of a socio- educational Program evaluation that supports higher educational paths for underprivileged students in Argentina. Specifically, for this article, we will analyze the reasons that beneficiaries of that program, drop out of it. The analysis is based on a qualitative methodology which consisted on 15 in depth interviews to scholars who quitted the Program, inquiring both who "decided" to drop out and those who were asked to do so by the Program authorities. This research shows current trends in educational paths, which combine periods of dropping out, with the promise of many times already accomplished- going back to university. Also, the need to make compatible studies and work.
\end{abstract}

Keywords: access to education, university students, dropping out, educational policy

\footnotetext{
1 Investigadora del Consejo Nacional de Investigaciones Científicas y Técnicas por la Universidad Nacional de Misiones, Argentina. Doctora en Ciencias Sociales de la Universidad de Buenos Aires, Argentina. Dirección electrónica: Izmayer@gmail.com

2 Becaria Doctoral del Consejo Nacional de Investigaciones Científicas y Técnicas en CITRA - Universidad Metropolitana para la Educación y el Trabajo, Argentina. Magister en Ciencias Sociales con Orientación en Educación de FLACSO Argentina. Doctoranda UBA. Dirección electrónica: cerezoleticia@gmail.com
}

Artículo recibido: $1^{\circ}$ de noviembre, 2017

Enviado a corrección: $1^{\circ}$ de marzo, 2018

Aprobado: 23 de marzo, 2018 


\section{Introducción}

La literatura respecto de la educación superior coincide al explicar el desarrollo histórico del sistema universitario, que el mismo fue creado para una elite (Bourdieu y Passeron, 2009; Buchbinder, 2005; Carli, 2012; Herrero, 2010) y luego, la procedencia social del estudiantado se fue modificando con la incorporación de estudiantes provenientes de sectores sociales medios, primero, y bajos, más recientemente. En tal sentido, el análisis respecto de este nivel educativo, es indisociable del de la desigualdad, es decir de las formas en que las instituciones educativas en sus diversos niveles en general y el superior en particular, participan en la producción o no de sociedades más justas e igualitarias.

En términos estructurales, es lícito afirmar que Argentina, así como varios otros países de la región, desde el comienzo del nuevo milenio, ha estado embarcada en el combate de las desigualdades sociales (Kessler, 2015), dentro de las que se encuentran las educativas (Dussel, 2005; Tiramonti, 2008). Al respecto cabe referir a las modificaciones de la legislación educativa ${ }^{3}$ que extendió la obligatoriedad de la educación a trece años, incluyendo la finalización de la escuela secundaria. Asimismo, la puesta en marcha de diversos programas sociales que buscan favorecer la inclusión y retención de los estudiantes en las aulas.

El proceso de ampliación de la matrícula, si bien presenta ciertas especificidades para el caso argentino, es parte de un fenómeno generalizado de occidente. Esta tendencia, que comenzó en los países centrales de occidente, fue desarrollándose también en los de América Latina (Ezcurra, 2009 y 2011; García de Fanelli y Jacinto, 2010; Pochulu y Williams, 2004). Así, el Instituto Internacional para la Educación Superior en América Latina y el Caribe (IESALC) de la UNESCO, indica que entre 1994 y 2006 la matrícula de la educación superior mostró una tasa de crecimiento sobresaliente del 126\%, pasando de albergar aproximadamente 7.544.000 estudiantes a alrededor de 17.017.000 (Gazzola y Didriksson, 2008). Esta matrícula cabe señalar, se concentró principalmente en los estudios de grado (96\%) y en instituciones de gestión pública gratuita. El caso argentino no quedó por fuera de

\footnotetext{
${ }^{3}$ Con el objetivo de garantizar la igualdad de oportunidades de aprendizaje, apoyar las políticas de mejora en la calidad de la enseñanza y fortalecer la investigación científico-tecnológica la Ley de Financiamiento Educativo 26.075 de 2005, establecía un incremento de la inversión en educación, ciencia y tecnología por parte del Estado Nacional y las 24 jurisdicciones argentinas, hasta alcanzar en el año 2010 una participación del 6\% en el Producto Bruto Interno. Luego, en 2006 se sancionó la Ley Nacional de Educación (№. 26.206) que reemplazó la Ley Federal de Educación. La misma, entre otros aspectos importantes, ordena el sistema educativo en cuatro niveles (educación inicial, educación primaria, educación secundaria y educación superior), establece las modalidades de la educación y establece la obligatoriedad del sistema desde la edad de 4 años, tras su modificación en 2014 producto de la sanción de la Ley 27.045, hasta la finalización del nivel de la educación secundaria.
} 
estas tendencias observando un gran crecimiento en la matrícula universitaria en los últimos años. En 1960 la matrícula universitaria argentina alcanzaba a 160 mil, al tiempo que se observa una tendencia creciente en su reclutamiento hasta alcanzar en el año 2013 a un poco más de un millón ochocientos mil estudiantes universitarios cifra que continúa incrementándose (Argentina, Ministerio de Educación, Secretaría de Políticas Universitarias, 2013) $)^{4}$

Sin embargo, la estrategia de ampliar sus bases no alcanzó para evitar la deserción y abandono de los estudios, principalmente durante el primer año de cursada. Tal como lo describe Ezcurra (2011) se generó un modelo de inclusión excluyente que consiste en una tendencia al fracaso académico y al abandono de los estudios universitarios en las franjas sociales que se encuentran en desventajas respecto de la distribución del capital económico y cultural, dando cuenta de las desiguales probabilidades de graduación entre los distintos sectores sociales. Proceso que tuvo lugar inclusive sin desmerecer la ampliación de la oferta educativa en nivel superior y las políticas inclusivas a nivel educativo desplegadas en los últimos años en Argentina (Ezcurra; 2011; Fernández Lamarra, 2002 en Carli 2012; García de Fanelli, 2005). En consecuencia, si bien el proceso de ampliación y masificación del sistema educativo en general y del acceso al nivel superior benefició a los sectores socioeconómicamente desfavorecidos, siguiendo el análisis de Tinto (2006), el abandono en las franjas sociales más bajas es mucho más alto que en las clases acomodadas e inclusive se estaría incrementando en los últimos años. En el caso particular de Argentina el éxito en la ampliación de la matrícula no se ha visto reflejado en la tasa de graduación: en el año 2013 el total de egresados de grado y pregrado de las 50 universidades de gestión estatal fue de 80.343 , cifra que representa el $25 \%$ de los nuevos inscriptos a esas casas de estudio $^{5}$. Si bien no es posible seguir la trayectoria de los estudiantes de forma individualizada, podríamos sostener que de cada 100 estudiantes que se inscribieron en la universidad 25 lograron egresar de las mismas. Al respecto Guadagni y Boero (2015) indican que de cada 100 estudiantes que ingresan en el sistema de educación superior universitario, 70 de ellos no se gradúan, enfatizando a partir de estas cifras en que uno de los desafíos de la universidad es mejorar los bajos niveles de graduación, particularmente los de las carreras científicas y tecnológicas.

\footnotetext{
${ }^{4}$ Estas cifras reflejan la matrícula total -universidades públicas y privadas- de Argentina. Para un análisis más exhaustivo de las proporciones y diferencias entre cada sector, véase Toribio, D. (2010) y Anuarios de Estadísticas Educativas de la Secretaría de Políticas Universitarias del Ministerio de Educación de Argentina.

5 Elaboración propia con base al Anuario 2013 de Estadísticas Educativas del Departamento de Información Universitaria de la Secretaría de Políticas Universitarias del Ministerio de Educación de la República Argentina.
} 
Analizando el peso relativo de los factores asociados a la probabilidad de dejar los estudios universitarios, García de Fanelli y Adrogué (2015) a partir de la Encuesta Permanente de Hogares $^{6}$ refieren que en la tasa global de abandono de los jóvenes de 18 a 30 años se observa una brecha importante según sector socioeconómico: el 55\% de los jóvenes que en el 2013 pertenecían a hogares de bajo ingreso per cápita había abandonado, cifra que se contrapone con el $25 \%$ en igual situación entre los de mayor ingreso per cápita.

Con el fin de modificar esta subrepresentación de los sectores más bajos en lo que refiere a la matriculación y asistencia a la educación superior por un lado y a las tasas de graduación por el otro, surgieron diversas iniciativas orientadas a favorecer la permanencia en la universidad y contrarrestar las tendencias a la deserción. Dentro de las iniciativas estatales más importantes, se encuentran el Programa de Calidad Universitaria de la Secretaría de Políticas Universitarias, los Proyectos de Mejoramiento de la Enseñanza y el Programa Nacional de Becas Universitarias, ambos impulsados por el Ministerio de Educación de la Nación Argentina y el Programa de Respaldo de Estudiantes de la Argentina -PROG.R.ES.AR- financiado con recursos del Tesoro Nacional, entre otros. Asimismo, distintas universidades han incorporado estrategias variadas con el objetivo de acompañar a los estudiantes en su trayectoria, entre las que es posible destacar las tutorías universitarias, estrategias de seguimiento periódico de sus estudiantes, programas de ingreso y permanencia, entre otras.

Desde el sector privado también se pusieron en marcha iniciativas similares aunque de menor escala. El artículo presenta parte de los hallazgos de una evaluación de resultados ${ }^{7}$ de un Programa de inclusión universitaria en las trayectorias de jóvenes en situación de vulnerabilidad social, financiado por una entidad bancaria de Argentina y gestionado por organizaciones de la sociedad civil (OSC) del campo educativo. Como veremos en detalle en el próximo apartado, el Programa en cuestión otorga financiamiento mensual a un grupo de jóvenes -becarios- y un sistema de tutorías, para apuntalar su tránsito en la universidad. Si bien se trata de una plataforma "exitosa" en lo que refiere a la retención de las personas

\footnotetext{
${ }^{6}$ La Encuesta Permanente de Hogares (EPH) es un programa nacional de producción sistemática y permanente de indicadores sociales que lleva a cabo el Instituto Nacional de Estadística y Censos (INDEC), que permite conocer las características sociodemográficas y socioeconómicas de la población; así como también las modificaciones de las mismas.

7 Una evaluación tiene como finalidad generar información, conocimiento y aprendizaje dirigidos a alimentar la toma de decisiones oportunas y pertinentes para garantizar los resultados de un Proyecto (SIEMPRO, 1999 en Fernández Prieto y Cerezo, 2010). Nirenberg (2010) sostiene que la evaluación de resultados contribuye al diseño y gestión de los proyectos sociales en tanto permite indagar, no sólo valorar los logros de la intervención, sino también respecto de los obstáculos emergentes, las posibles mejoras y/o correcciones en la intervención.
} 
becadas participantes, presenta también casos en los que, o bien por decisión institucional o bien decisión de los jóvenes, su participación en el Programa finaliza antes de la graduación. En las líneas que siguen, presentaremos las características del Programa y la estrategia metodológica de la investigación, y analizaremos luego cuáles son los repertorios que forzaron su salida del Programa, ya sea por decisión propia o institucional.

\section{Características del Programa analizado}

El Programa de inclusión universitaria estudiado es implementado desde el año 2008 en la Argentina por la Gerencia de Responsabilidad Social Corporativa de una entidad financiera local, para colaborar con la educación superior de jóvenes de 17 a 23 años en situación de vulnerabilidad social a través de un apoyo económico -estipendio mensual ${ }^{8}$ - y del seguimiento de la trayectoria y rendimiento universitario - a través de la implementación de tutorías ${ }^{9}$ - que se desarrolla en convenio con diferentes OSC de forma externa a las instituciones educativas. Tanto el estipendio como el pago a los tutores son financiados por la entidad bancaria responsable, al mismo tiempo que delimita las condiciones de ingreso y permanencia en el Programa $^{10}$. El contacto cotidiano de los becarios es con las organizaciones que gestionan su beca que deben informar su rendimiento académico a la entidad bancaria. Hasta el año 2012, año en que comienza la evaluación y se realiza el trabajo de campo, nueve OSC se encargaban de la selección de las personas becadas, de la gestión y coordinación de las becas, alcanzando en total a 151 jóvenes que se distribuyen territorialmente en diversas jurisdicciones argentinas, con una fuerte preeminencia en el Gran

\footnotetext{
${ }^{8} \mathrm{Al}$ año 2012 el estipendio para los primeros dos años de estudio constaba de $\$ 850$ (equivalente a USD 177) y para los cuatro años siguientes descendía a $\$ 650$ (USD 135). Esta diferencia se debe a que el Programa habilita a las personas becadas a trabajar a partir del segundo año de cursada.

${ }^{9}$ Las tutorías universitarias son prácticas socio-educativas que pretende facilitar, mediar, acompañar y orientar a los estudiantes en la construcción de un lazo social con la institución educativa. Constituyen repertorios institucionales (Garcia de Fanelli, 2005) que pueden contribuir en la trayectoria educativa. Estas prácticas son plausibles de tomar diversas modalidades, hay quienes sostienen que los tutores dan apoyo, seguimiento y orientación a los estudiantes que están en proceso de adaptación a la institución educativa (Jacinto y Terigi, 2007), o bien quienes sostienen que las tutorías consisten en generar interacción y vinculación del tutor en la trayectoria educativa de los estudiantes (Urresti, 1999).

${ }_{10}$ Respecto de los criterios para ingresar al Programa los jóvenes debían acreditar, al momento de aplicar: a. tener recursos económicos limitados; b. no poseer otros subsidios (becas) para su educación superior; c. no estar trabajando al momento de solicitar su inclusión en el Programa; d. tener oportunidades limitadas por su entorno social y geográfico. Por otro lado, los aspirantes tenían que estar inscriptos en alguna de las siguientes carreras en universidades nacionales: Informática y todas sus derivadas; Administración de Empresas; Economía; Contador Público; Comercio Exterior; Ingeniería Industrial; Ingeniería Agrónoma. La permanencia en el Programa exige 4 materias aprobadas por año.
} 
Buenos Aires (GBA) ${ }^{11}$ - $37 \%$ en Ciudad de Buenos Aires y $25 \%$ en los partidos del Gran Buenos Aires. El $27 \%$ restante está distribuido a lo largo y ancho del país, destacándose la cantidad de becarias y becarios de las provincias de Neuquén, Mendoza y Jujuy ${ }^{12}$.

\section{Estrategia metodológica de la investigación}

Los objetivos de la evaluación cuyos resultados expondremos fueron evaluar los resultados del Programa en las trayectorias educativas de jóvenes que forman parte del mismo; así como también dar cuenta de los motivos por los cuales algunos abandonaron el Programa.

Cabe introducir aquí qué entendemos por trayectorias educativas. Esta noción nos permite articular en un mismo concepto las elecciones de los sujetos, las experiencias familiares, las propuestas institucionales disponibles, el contexto social que opera condicionando los recorridos y las prácticas (Montes y Sendón, 2006 y 2010). El concepto enlaza las prácticas y las estrategias desplegadas por los agentes, así como también los elementos estructurales de los grupos sociales y de los individuos que son puestos en acción en un determinado contexto, entre ellos los capitales culturales, económicos y simbólicos (Tiramonti, 2008). Terigi (2007) distingue entre trayectorias educativas teóricas o ideales, siendo estas las que expresan itinerarios en el sistema que siguen la progresión lineal prevista por éste en los tiempos marcados por una periodización estándar; de las reales, es decir las que reconocen tanto itinerarios frecuentes o más probables como recorridos que no siguen ese cauce. Esta diferenciación nos hace posible matizar el concepto en función de las heterogeneidades existentes en los itinerarios de los estudiantes.

Con la intención de abordar la trayectoria educativa tanto de personas becadas y "ex becarios" desde su propia experiencia y los modos en que se relacionan con el Programa y sus agentes institucionales, se optó principalmente por una perspectiva cualitativa. Esta elección refiere a que las mismas hicieron posible acceder al universo de significaciones de los actores, así como también dilucidar estructuras y argumentos conceptuales complejos sobre las que se basan las prácticas y creencias de los agentes (Long y Long, 1992 en

\footnotetext{
${ }^{11}$ El Gran Buenos Aires (GBA) es definido por el Instituto Nacional de Estadísticas y Censos (INDEC) como el área comprendida por la Ciudad de Buenos Aires (CABA) más los Partidos del Gran Buenos Aires, que son 24 partidos completos que se incluyen en la definición en su totalidad. (INDEC, Censo 2010).

12 Esto se debe a los convenios y acuerdos firmados entre la entidad bancaria y las OSC por un lado y a la oferta universitaria por el otro.
} 
Mayer, 2012) ${ }^{13}$. De allí que la evaluación consistió en la realización de nueve grupos focales $^{14}$ a jóvenes integrantes del Programa por OSC y dieciséis entrevistas de profundización a algunos jóvenes participantes de los grupos focales según variables definidas por las investigadoras ${ }^{15}$. Por otra parte, a modo de recolectar información cuantitativa, se trabajó con encuestas autoadministradas para complementar los datos cualitativos. Luego, para el segundo objetivo mencionado, se entrevistó ${ }^{16}$ a un total de 15 ex becarios del Programa. El análisis que presentamos en este artículo hace uso específico de estas entrevistas, no así del resto de las técnicas implementadas. Estas entrevistas

13 En todos los casos de la investigación -becarios y ex becarios- sus repertorios fueron abordados comprensivamente, es decir, teniendo en cuenta los contextos específicos dentro de los que los mismos se desarrollan. Desde nuestra perspectiva, todo actor social al narrar una situación determinada, la produce. En relación a nuestro diseño metodológico, coincidimos con Saltalamacchia cuando señala que el individuo es "...un lugar de "anudamiento" de un conjunto determinado de relaciones sociales" (1992, p. 38), por ello es de suma importancia acercarnos al conocimiento de las trayectorias de los actores como observable de los sistemas relacionales en los que se encuentran insertos. En este sentido, creemos que cada entrevista no estructurada puede ser considerada un verdadero testimonio de la situación que experimentó el entrevistado. Como sostiene Saltalamacchia (1992), por definición, todo entrevistado es un actor de los sucesos narrados: lo vivió y en su relato los interpreta, tanto desde intereses materiales o simbólicos que organizaron su participación, como por instrumentalidades cognitivas. Entonces, los datos que provee, deben ser evaluados o tenidos en cuenta, incorporando esa construcción.

${ }^{14}$ La opción por esta técnica se debió a los objetivos de la evaluación. Por tratarse de una estrategia cualitativa, esta técnica permitía obtener información del Programa, a través de un debate respecto al mismo. De esta manera, los grupos focales constituyeron espacios de reflexión de temas en común de la plataforma programática, su inclusión en ella, el rol de los agentes institucionales, posibles mejoras, etc. Cada grupo contó, en promedio, con 9 participantes. En el caso de las dos OSC más numerosas, se realizaron dos grupos focales en cada una de ellas, dividiendo a sus participantes por género. En el resto de la muestra, los grupos fueron mixtos.

${ }^{15}$ La investigación supuso una estrategia descendente, es decir desde los lugares de mayor poder hacia abajo. Con esto referimos a que primero, la entidad financiadora del Programa envió una carta institucional a los coordinadores de cada OSC, notificando la evaluación a realizarse y sus objetivos principales y a modo de presentación del equipo de trabajo. En la misma se solicitaba colaboración en la investigación y los requerimientos metodológicos. Una vez enviada esta nota, desde la coordinación del equipo evaluador se contactó a los referentes de las OSC y se programó una entrevista individual que suponía por un lado, la ampliación de la información respecto de la evaluación y diagramar los grupos focales con las autoridades, por el otro. Cabe señalar que en la mayoría de los casos eran ellos quienes convocaban a las entrevistas grupales a los y las becarias que se realizaron, casi en su totalidad, en las instituciones en las que se desempeñaban. Como ya fue mencionado, si bien fueron los investigadores quienes establecieron las variables y criterios de participación en los grupos, al realizar las autoridades de cada institución la convocatoria y selección de las personas becadas, pudo imprimir un sesgo en los mismos. En el caso de las entrevistas de profundización, la selección corrió por parte del equipo evaluador, en función a la riqueza que ese caso podría aportar.

${ }^{16}$ En ambos casos, las entrevistas fueron no estructuradas, entendiendo por éstas aquellas en la que existe un planeamiento previo acerca de los temas a tratar pero que se puede modificar durante el transcurso de la entrevista misma. En las mismas se indagó y profundizó en las trayectorias de los actores para aprender los cambios en la posición estructural de los mismos y el desarrollo de su experiencia social, tratando de captar las creencias y las sensibilidades políticas particulares de estos individuos. Nos interesa acercarnos a la perspectiva del actor en su contexto abandonando el punto de vista "único" en beneficio de la pluralidad de puntos de vista coexistentes (Bourdieu, 1999). Como es lógico, las investigadoras aportaron a las entrevistas sus instrumentos analíticos, su entrenamiento en el análisis de situaciones y un conocimiento quizás más global sobre el proceso; conocimiento logrado tanto mediante el previo estudio de fuentes secundarias como de otras entrevistas. Pero, una vez en la relación, ese bagaje es sólo uno de los insumos. La entrevista debe ser entendida como el marco de una elaboración teórica conjunta en la que no necesariamente surgirán acuerdos totales o aprovechamientos semejantes; pero sí interpretaciones que no existían antes de la relación (Saltalamacchia, 1992). 
supusieron un gran trabajo de localización, ya que en primer lugar, varias de las OSC participantes habían perdido el contacto con estos jóvenes por diversos motivos (falta de sistematización de casos, cambio de autoridades, etc.) y otras propias de los jóvenes en cuestión, como mudanza de ciudad, cambios de datos de contacto, etc. Sin embargo, a medida que se fue desarrollando el trabajo de campo, accedimos a varios de los jóvenes que ya no pertenecían al Programa al momento de realizar la investigación. Por otra parte, el acceso a este último grupo -los "ex- becarios"- contó con otra dificultad adicional: mientras que para el caso de las personas becadas que participaban activamente del Programa al momento de la evaluación, la participación contó con ciertos grados de obligatoriedad, para el caso de quienes por diversos motivos ya no formaban parte del mismo, la participación era completamente voluntaria, es decir que la posibilidad de entrevistarlos y acceder a ellos dependía de su deseo -o no- de relatar su experiencia.

En el caso particular del subgrupo de ex becarios, las entrevistas se refieren a un proceso finalizado, de los que la persona conoce el desenlace y posiblemente, "sufre" alguna de sus consecuencias. En este contexto, la memoria de los hechos está signada por los hechos y secuencias recordadas. En este sentido, nos interesa hacer hincapié en las capacidades reflexivas de los actores por cuanto se orientan hacia los esfuerzos de sus relatos. El registro reflexivo se identifica como una propiedad de la acción de estos actores, que conforma de manera definitoria las características de la misma ${ }^{17}$.

En todos los casos, al momento de comenzar las entrevistas o grupos focales, se advirtieron los objetivos de la investigación, al tiempo que se aseguró el anonimato para las publicaciones posteriores, para lo cual o bien sus nombres fueron ficcionados o sólo sus fragmentos delimitados por datos sociodemográficos (género y edad).

\section{4. ¿Quiénes se fueron?}

Los casos de jóvenes que dejaron el Programa analizado, constituyeron de gran interés y utilidad para la investigación en tanto nos posibilitaron aproximarnos a los motivos

\footnotetext{
17 Según Giddens, la reflexividad es "la forma específicamente reflexiva del entendimiento de agentes humanos la que interviene a mayor profundidad en el ordenamiento recursivo de prácticas sociales. Una continuidad de prácticas presupone reflexividad, pero la reflexividad misma sólo es posible en virtud de la continuidad de prácticas, que las define claramente como "las mismas" por un espacio y un tiempo. "Reflexividad", entonces, no se debe entender como mera "auto-conciencia" sino como el carácter registrado del fluir corriente de una vida social. [...] El registro reflexivo de la actividad es un rasgo permanente de una acción cotidiana, que toma en cuenta la conducta de un individuo, pero también la de otros. Es decir que los actores no sólo registran de continuo el fluir de sus actividades y esperan que otros, por su parte, hagan lo mismo; también registran por rutina aspectos sociales y físicos de los contextos en los que se mueven." (Giddens, 1998: 40-41, 43).
} 
por los que concluyeron su participación en el mismo. En las próximas líneas daremos cuenta de los repertorios que explican la salida del Programa de los "ex becarios", dividiendo los mismos en dos grandes grupos: quienes salieron del Programa por sugerencia de las OSC a través de las cuales se materializaba su adhesión a la estrategia y quienes se "salieron" por iniciativa propia.

Antes de pasar a detallar cada uno de estos repertorios, resulta pertinente realizar una salvedad, relacionada con la diferencia entre dejar el Programa y abandonar la universidad. Al momento de realizar el trabajo de campo, con la excepción de uno, todos los "ex becarios" entrevistados continuaban sus estudios universitarios, ya sea la misma carrera seleccionada inicialmente o bien otra carrera. Sólo un caso no se encontraba estudiando, pero con el proyecto de retomar la universidad al año siguiente, es decir que si bien habían finalizado su participación en la estrategia no habían puesto fin a su trayectoria universitaria.

\subsection{Apartamiento del Programa por decisión institucional}

En este apartado, analizaremos los casos en que las personas becadas fueron apartadas de la plataforma por decisión -consensuada o unilateral- por la OSC gerenciadora de su beca. En tal sentido, analizaremos la significación que las y los jóvenes les dan a las decisiones y a las narrativas institucionales.

\subsubsection{No alcanzar los requisitos y exigencias mínimas}

La permanencia en el Programa contaba como requisito aprobar 4 materias anuales. Dentro de este primer subgrupo, reunimos a aquellas personas becadas que debieron abandonar el Programa por no cumplir con este requisito de rendimiento académico que supone para su permanencia. Esto aparece en el relato de una ex becaria:

Investigadora: ¿Y por qué dejaste de pertenecer a [nombre del Programa]

Becaria: Por el tema de las materias. Viste que te pedían 4 materias mínimo por año. Entonces yo en una no llegué, fallé digamos. Dos había metido en el año y otra que después ya me había ido mal entonces... por eso.

I: ¿Ellos te dijeron o vos decidiste irte?

B: No, no, ya una vez me la habían perdonado, dos veces ya no te perdonan, digamos. Es una cosa así. No podes digamos, volver a repetir eso.

I: ¿O sea que ya no cumpliste con los requisitos?

B: Claro, era uno de los requisitos. Entonces... es por eso, no es que dije bueno abandono y chau. (Mujer, 21 años, GBA) 
Este fragmento nos advierte de una reincidencia en el incumplimiento de los requisitos por parte de la ex becaria. Entonces, debemos enunciar dos aspectos: que su separación del Programa no es inesperada, ya que había tenido "advertencias" por un lado, y por otra parte, que pese a tener conocimiento de esas limitaciones, la joven no pudo cumplir con los requisitos para permanecer dentro de la estrategias de inclusión universitaria. Esto nos permite hipotetizar que existen personas becadas - aun cuando se presenten minoritariamente- que no logran alcanzar los requerimientos de permanencia del Programa. En este caso, no parece haber un desinterés, sino que se trata más bien de un impedimento, ya sea por su realidad individual y familiar, por la distancia existente entre las prácticas y la cultura universitaria que demandan las instituciones educativas, por la ausencia de estrategias desplegadas por las instituciones universitarias y sus actores que apuntalen las trayectorias educativas de los estudiantes, así como también una combinación de los aspectos anteriores. Todo ello vuelve, en términos de Terigi (2007), su trayectoria real incompatible con la trayectoria teórica propuesta y deseada por el Programa.

La salida del Programa se da, desde esta perspectiva, por incumplimiento de los requisitos exigidos, enfatizando en la responsabilidad individual de la persona becada, en particular porque estaba al tanto de esas reglas de permanencia y no alcanzó a cumplirlas. La misma ex becaria cuenta cómo fue que se enteró de su apartamiento:

Becaria: Me lo dijo la coordinadora...

Investigadora: ¿Ella te dijo que no ibas a poder seguir por ese motivo?

B: Sí, porque era muy obvio, ella me dijo... yo no iba a poder cumplir con los requisitos, entonces... ya no me iban a informar más, digamos. La coordinadora una vez me dijo vení a firmar unos papeles, o sea como diciendo ya está o por el lado de que yo dije bueno dejo la beca, pero no sabía si decirle dejar o que ya no me la den porque era lo mismo digamos prácticamente, la beca ya la había perdido.

I: ¿Por qué?

B: Sí, porque si te dan una oportunidad, la segunda oportunidad, ya no la, te fue mal, yo por ese lado lo tomé como ya está, entonces...

I: ¿Lo diste por hecho?

B: Claro, lo di por hecho, entonces como que bueno ya está, me dijo vení a firmar unos papeles, y una vez fui porque yo estaba haciendo un curso en otro lado, por el tema de la AFIP y el gobierno, y fui y no estaba. Ya no estaba porque resulta que entraba a las tres de la tarde. $Y$ bueno dije, no, yo me tenía que ir a otro lado, 
entonces este verano agarré y bueno le dejé a la persona que estaba ahí y le dije mira “¿le decís que yo vine?”, y nada, después ella me llamó y me dijo venite un día. Y justo esos días tampoco podía por el tema de la facultad, mis horarios no coincidían digamos, entonces bueno, agarré y lo dejé pasar, lo dejé pasar, entonces yo le mandaba emails pero no me contestaba, de las dos veces que envié no me contestaba, después volví a llamar una vez, me contestó una chica, me dijo mirá no está, Ilamá a la tarde. Volví a llamar... no, no está porque se había ido de viaje. Y así. Así que no la pude ubicar. O sea, al final no firmé el papel, pero calculo que no... Eso fue todo... (Mujer, 21 años, GBA)

Es importante resaltar, tal como lo vemos en el fragmento de la entrevista anterior, que hubo un aviso previo, una alerta respecto de su permanencia en el Programa, pero advirtiendo que esta situación no podía reiterarse. Tal vez lo que podemos preguntarnos ante estos casos refiere a los dispositivos puestos en marcha -o no- por las instituciones que gestionan las becas para que estudiantes alcancen los objetivos planteados en los documentos programáticos. En este sentido, algunas de las OSC participantes incluían talleres, clases particulares con voluntarios que conocían los contenidos a rendir, entre otras estrategias para apuntalar las trayectorias universitarias, mientras que otras se limitaban a ofrecer el estipendio y un sistema de tutorías más o menos intenso (Mayer y Cerezo, 2014). Inclusive, algunas organizaciones conversaban con la entidad financiadora casos especiales donde convenía "aflojar" los requisitos, mientras otras, con casos en similares situaciones, se limitaban a acatar las normas, sin indagar en las cuestiones específicas que hacían al no cumplimiento de los requisitos pautados. Pareciera existir aquí cierta tensión entre dos posturas, al menos en función de los casos problemáticos: la adhesión a la norma, o su flexibilización al menos en determinados grados. Contextos familiares adversos, tareas dentro del hogar y trabajos no remunerados asociados comúnmente al cuidado de familiares enfermos o menores, por ejemplo, son algunos ejemplos de situaciones que pueden derivar en hacer más flexibles los requisitos pautados, no por falta de compromiso con el estudio ni desinterés, sino porque bajo determinadas circunstancias, es imposible alcanzarlos. La adhesión a la norma, plantea una estandarización y supone que todas las personas becadas comparten el mismo punto de partida, cuando en realidad se trata de colectivos diversos que presentan situaciones heterogéneas muchas veces, dignas de consideración. 
Flexibilizar ciertas reglas ante casos especiales puede traer consecuencias sobre las que vale la pena reflexionar. Esta evaluación queda supeditada al esfuerzo que realice o no cada OSC, al interés o no en la permanencia de sus becarios y becarias dentro del Programa. Esto supone gestiones específicas -reuniones, comunicaciones por diferentes vías, etc.- que le agregan tareas a las ya definidas e irrenunciables del Programa. Luego, también podemos pensar que estos arreglos "excepcionales" y particulares pueden desembocar en situaciones de malestar entre el resto de las personas becadas, que sí cumplen con los requisitos y seguramente, en muchos casos pueden llegar a considerar que serían también merecedores de tal flexibilidad.

Ligado a lo anterior, nos interesa poner en consideración, para este caso en particular pero que puede extrapolarse a otras experiencias educativas, que los márgenes de flexibilidad, si bien posibilitan reconocer que existen puntos de partida diferenciales, tal reconocimiento, puede permitir puntos de llegada similares. Por otra parte, también plantea el interrogante de hasta dónde es justo flexibilizar o dicho de otra manera, qué casos atender y cuáles no, cómo no caer en una extrema condescendencia o en situaciones injustas para el resto de la población objetivo. De no tener en claro los límites a estas digresiones, se plantea el peligro de tornar un Programa con ciertas reglas, recursos, derechos y obligaciones en otro de "plastilina" (Tenti Fanfani, 2007) formateado más por su público que por los agentes institucionales que lo diseñan, implementan y gestionan y en donde el cumplimiento de las normas puede terminar siendo la excepción.

\subsubsection{Cambio de carrera}

Aquí se trata de cambios en las orientaciones de estudio de las personas becadas de este Programa, en donde se registró un viraje hacia carreras no reconocidas como "financiables" por la entidad bancaria. En términos de Tinto (1989) estas modificaciones son denominadas deserciones voluntarias en tanto son las que tienen que ver con la falta de coincidencia entre los planes de los individuos, ya sean iniciales o generados en el transcurso de sus trayectorias, con las propuestas de la universidad. Dentro de este tipo de deserción universitaria se incluyen las dudas "vocacionales" así como también las intenciones de cambiar de carrera.

Muchas de las OSC que participan de esta estrategia, gestionaban al mismo tiempo, becas de programas similares financiados por otras entidades lucrativas del país. De modo que en estos casos no se corrobora un proceso de desafiliación, es decir que las personas 
becadas continúan bajo el radar de la misma OSC, pero cambiando de financiador, hacia otro sin requisitos para los nuevos estudios elegidos o donde esa carrera no presentaba inconvenientes.

\subsection{Abandono por decisión del becario}

En los apartados siguientes, describiremos los repertorios que dan cuenta de los distintos motivos por los cuales un grupo minoritario de jóvenes decide abandonar el Programa. Huelga aclarar que la enumeración de estos repertorios responde a divisiones y abstracciones analíticas que nos permiten ordenar conceptualmente la realidad, al dar cuenta de las justificaciones de los jóvenes para legitimar su salida de las estrategias de inclusión universitaria. Dicho de otra manera, un joven puede adherir a uno, varios o inclusive todos los repertorios enumerados a continuación. Por último, y en relación a esto, debemos aclarar que si bien los justificativos aparecen de manera individual son a la vez expresiones de situaciones colectivas.

\subsubsection{Altruismo}

El primer argumento que aparece es el del "altruismo". Este consiste en considerar que en la medida que los recursos son limitados que, otro joven podría utilizar mejor los recursos. En los casos en los que esto se combina con una recomposición económica del tejido familiar primario, aquí el altruismo se conjuga con el reconocimiento objetivo de una "no necesidad" de la prestación económica. Vale la pena aclarar que, mediante este concepto, no nos referimos a que los jóvenes que adhieren a estos repertorios sean "buenos", una tendencia que puede soslayarse en los análisis de Ghilardi y Spallarossa (citado en Mayer, 2012) o en las conceptualizaciones de Duschatzky (1999), sino que mediante este concepto nos referimos a los argumentos que enuncian los agentes para enunciar que sus prácticas son las más favorables para ciertas situaciones y además, parte de una definición realizada por ellos mismos sobre quiénes "merecen" estar dentro del Programa. Como lo afirma un ex becario:

"Era sacarle un año más de plata que yo al Banco, prefería darle mi lugar a alguien que lo necesitará porque yo estaba muy bien y les estaban dando bastante prioridad a los chicos del interior, que con la plata que le daban hacian malabares porque eran increíbles las cosas que podían hacer, yo veía que les servía así que me pareció que mi lugar por ahí lo aprovechaba más otro." (Varón, 20 años, GBA) 
Aquí el becario se manifiesta solidario con sus pares por un lado, al tiempo que como veremos a continuación, reconoce que ya no le hace falta la beca:

“Lo dejé yo, era la baja voluntaria, fui a hablar con... ahí en [Nombre de la OSC]. Le comenté que había empezado a trabajar y que no iba a cumplir con los requisitos, le dije que me encantó, me ayudó un montón porque me daba mis gustos. Las charlas me gustaron, ir a la torre de Banco, nos daban charlas, nos juntábamos con los chicos, compañeros míos que también estaban becados.”(Varón, 20 años, GBA)

Las narrativas incluidas en este grupo, responden también a cierta lógica de filantropía social que existe en el marco del Programa. Ya desde su propia enunciación, el mismo se propone colaborar con jóvenes que pese a condiciones de vida desfavorables, muestren aptitudes o talentos que los destaquen de otros jóvenes que se encuentran también en condiciones de vulnerabilidad social. En tal sentido, si bien se enfatiza en las carencias socioeconómicas, la desigualdad social por sí sola no sería un marco habilitante para "ser parte" del Programa: para integrar la estrategia hay que merecerlo y esto se asimila a esforzarse y destacarse del resto en términos de rendimiento académico. Esta dimensión, que está presente en los documentos programáticos, se refuerza con acciones específicas desarrolladas por la entidad financiera, como por ejemplo premiar a los mejores promedios de cada año. Estas acciones plasman el modo en que desde el Programa se define quienes pueden ser sus destinatarios y quienes no, siendo personas beneficiarias de la estrategia aquellas que "merece" ser parte y comprendiendo a los dispositivos diseñados como beneficios, no derechos.

\subsubsection{Exigencias académicas inalcanzables}

En el apartado anterior hemos desarrollado que, en algunos casos, eran las propias OSC las que, al advertir que no cumplían con los objetivos programáticos, tomaban la decisión de suspender o cancelar la participación del becario en el Programa. Sin embargo, en la mayoría de los casos en los que se registra el abandono por este motivo, la misma es consecuencia de la decisión de los jóvenes. Aquí, al igual que lo sucedía anteriormente, los jóvenes no pueden alcanzar los objetivos propuestos por el Programa y deciden entonces, abandonarlo, luego de no haber cumplido con esos requisitos al menos una vez y conversado esto con los agentes institucionales correspondientes. En muchos casos, más allá de las posibilidades que las OSC les propongan, como, por ejemplo, pedir 
excepcionalmente a la entidad bancaria que mantenga la beca pese a no cumplir con los objetivos programáticos, las personas becadas deciden no continuar dentro de la plataforma pero sí con sus estudios universitarios. Nuevamente, la exigencia por mantenerse en el Programa se torna negativa, o inclusive asfixiante para los que no pueden alcanzar los objetivos. La decisión es entonces, salirse del Programa pero no dejar de estudiar en la universidad, sino continuarlo con sus ritmos particulares. En palabras de los jóvenes, dejan el Programa porque "voy haciendo la facu como puedo" o bien porque "hago la carrera más tranquilo."

De alguna manera, y aun cuando reconocen la importancia de la dimensión colectiva en la que estaban insertos, son las mismas personas becadas quienes deciden seguir por su propia cuenta ajustando su trayectoria universitaria a sus tiempos y posibilidades reales.

Si bien es cierto que el Programa supone ciertos requerimientos académicos mínimos que en estos casos aparecen como un factor de presión, es justo señalar que aun cuando el sistema de educación superior vislumbre tendencias hacia su democratización -al menos cuantitativas- la universidad, en tanto dispositivo no ha logrado modificar sus propios mecanismos de reproducción, ni varios de los patrones culturales de discriminación sobre los que se asienta, como su ethos meritocrático, por lo que aun cuando muchas veces se lo proponga, continúa reforzando las desigualdades educativas y socioeconómicas. Como sostiene Sverdlick (2013), los sectores desfavorecidos de la sociedad muchas veces no llegan a la universidad porque su propio imaginario ha desterrado los estudios superiores de su trayectoria vital o porque sus realidades laborales no se lo permiten, pero inclusive cuándo lo hacen, la mayoría de ellos no consigue permanecer ni realizar sus estudios según las trayectorias ideales siguiendo los itinerarios académicos planteados. Más allá de las falencias de su formación en niveles de enseñanza anteriores que ameritan mencionarse, existe otro repertorio de dificultades relacionados con las carencias que tienen las propias universidades para garantizar el aprendizaje y permanencia de este tipo de estudiantes, como los horarios, el sistema de correlatividades y la desacreditación y desconocimiento de saberes "no científicos" que refiere a otras formas de sociabilidad y que remiten a universidades que siguen pensándose para un estudiante se puede acomodar más o menos libremente a su formato y prácticas curriculares. $Y$ si bien las becas, las tutorías y demás dispositivos implementados pueden mostrar ciertos niveles de éxito, no alcanzan para contrarrestar en su totalidad estas dificultades que se generan por la matriz propia del sistema de educación superior. 
En este punto, es importante retomar la idea del "compromiso" que supone aceptar participar de este Programa, financiado por una entidad claramente visible. Como sostienen Beech y Barrenechea (2011), cada vez más instituciones sin fines de lucro y compañías lucrativas aumentan su participación en proyectos sociales o caritativos que en una particular conjugación con las instituciones estatales, generan una particular asociación entre lo público y lo privado. Lo novedoso de estas acciones filantrópicas es que los financiadores tienen expectativas respecto de ciertas formas de "reintegro" de sus donaciones, como por ejemplo, que las personas becadas puedan trabajar en el Banco o elijan esta entidad para bancarizarse. Ball (2010, citado en Beech y Barrenechea, 2011), llama a esto filantrocapitalismo, aludiendo a las contraprestaciones o compromisos que los beneficiarios adquieren a futuro como reconocimiento al esfuerzo de las organizaciones en cuestión. Esto puede reforzar la inseguridad de las personas becadas en alcanzar los compromisos que el Programa supone, no sólo en el momento de ser becados, sino también a futuro, y por eso, elegir seguir "solos".

\subsubsection{Priorizar el trabajo}

Una dimensión que es complementaria a la idea de seguir estudiando según los tiempos individuales y posibilidades de cada ex becario, es la de trabajar. Sin duda, la imposibilidad de tener un trabajo según los requisitos del Programa aparece como una restricción fundamental para la continuidad dentro del mismo, en particular cuando el estipendio mensual que reciben en el marco del Programa no es suficiente para adquirir los recursos necesarios para el desarrollo de la cotidianeidad de la trayectoria universitaria, ya sean de tipo estudiantil directas (fotocopias, libros, materiales para estudio, viáticos para ir a sus lugares de estudio) o indirectas, referidas a los usos que relacionados con la posibilidad de permanecer en situaciones sociales y de estudio o académicas gracias al estipendio, o no estar pendientes de la plata para ver si pueden permanecer en una reunión académica o social (Mayer y Cerezo, 2016).

Como ya lo desarrollamos líneas arriba al describir las características principales del Programa, los jóvenes seleccionados no pueden trabajar durante los dos primeros años, al tiempo que reciben un estipendio que alcanza para los gastos básicos que un estudiante puede tener: viáticos, fotocopias, materiales didácticos y de trabajo, etc. En la mayoría de los casos y tal como lo hemos analizado en otros lugares (Mayer y Cerezo, 2016) las personas becadas logran posponer su deseo o necesidad de trabajo con este estipendio, asumiendo 
que el sacrificio de recibir menor cantidad de dinero les permite realizar la carrera "más rápido" o en otros términos, según la trayectoria ideal (Terigi, 2007), más tranquilos y coadyuva a buenos resultados académicos. Sin embargo, existen otros casos donde esto no sucede, y la posibilidad de trabajar, en particular cuando "aparecen" oportunidades, es irrenunciable. Las ventajas de trabajar al tiempo que estudian, según los ex becarios son muchas.

En primer lugar, existe una condición especial respecto de la universidad de gestión pública. No se trata sólo de que es gratuita sino que permite diferentes tiempos y dedicaciones a la cursada, lo que reconoce diferentes formas de ejercer el "oficio de estudiante" (Coulon, 2005), que no necesariamente coinciden con los requisitos para ser becario.

Por otro lado, existe un alto grado de confianza en la presencia de las instituciones educativas, no sólo en tanto que los anteceden, sino que estarán "siempre alli" y por lo tanto sus trayectorias universitarias pueden combinar períodos de cursada y trabajo con otros de sólo trabajo, configurando otros tipos de trayectorias universitarias, trayectorias reales, más vinculadas a sus realidades que las requeridas para sostener la beca.

Por otra parte y ya en el plano personal, aparece la estabilidad y con ella la posibilidad de planificar un futuro:

"Eso es lo que me gusta lo que tengo ahora con el trabajo, al saber que ya tengo una estabilidad, la carrera la hago más tranquilo. Tengo toda la vida por delante, me pongo a escuchar anécdotas de gente que se recibe a los 30 y pico sin embargo está todo bien." (Varón, 20 años, GBA)

La estabilidad se manifiesta de diversas maneras, no sólo en el cobro de haberes sino también en las condiciones de contratación. Los y las ex becarios/as valoran mucho los derechos que se desprenden de sus actividades laborales, entre ellos la cobertura de salud y los aportes jubilatorios.

Ligado a lo anterior, aparece también un espacio privilegiado asignado al trabajo frente a la trayectoria educativa "ideal" propuesta por el Programa y entonces abandonarlo se relaciona con la dimensión estrictamente económica. Mientras que el estipendio sólo alcanzaba para cubrir los gastos básicos de los jóvenes en tanto estudiantes, sus salarios les permiten además o bien ayudar a sus familias o bien acceder a bienes que de otra manera no podrían hacerlo. 
“El auto es mío, le puse un equipo a gas. Yo ahora me lo puedo costear con el trabajo. A mi mamá también la ayudó con las cuentas de la casa." (Varón, 21 años, GBA)

En este sentido, de acuerdo a la bibliografía al respecto (Beck, 2001; Castel, 1997), en los casos de ex becarios aparecen narrativas relacionadas a lo constructivo y habilitador que es la actividad laboral remunerada y aparece como una de las principales ventajas del alejamiento del Programa. En palabras de una ex becaria:

Investigadora: ¿Tu trabajo actual es temporario, sólo por el verano?

Becaria: Sí, por ahora sí, ahora que terminé con el tema de la facultad y de rendir y eso sí estoy trabajando para poder juntar un poco de plata, para mí, para lo personal, o sea... qué se yo un poco de, en las vacaciones también viene bien. (Mujer, 21 años, GBA)

No obstante, es importante resaltar que si bien los ex becarios le otorgan mucha importancia el trabajo para su cotidianeidad y actualidad, en algunos de ellos el estudio continúa apareciendo como importante, por lo que intentan no relegarlo o dejar la universidad por completo. Tal como refiere uno de los entrevistados al consultarles la importancia que le asignan a la educación y al trabajo:

“70\% a educación y 30\% al trabajo, el trabajo es más que nada por la plata, tampoco es una cosa que me interesa bastante. Y la educación es algo que me serviría para el día de mañana, pero ahora no tengo la posibilidad de que mis padres me paguen los estudios, por eso mismo tenía lo de la beca, si yo puedo estudiar y trabajar sería genial, pero me parece mucho más importante estudiar, por eso quiero seguir estudiando." (Varón, 20 años, GBA)

En este sentido, es importante resaltar que o bien los ex becarios continuaban sus estudios al momento de ser entrevistados, o como lo manifiesta el joven arriba citado, pensaba continuarlos para el comienzo del año lectivo siguiente, según lo afirmó en otros fragmentos de la entrevista.

Otro ex becario, también sostiene que deseaba trabajar y que por eso abandonó el Programa:

"Estuve tres años en el Programa pero después me dieron ganas de ir a trabajar. Yo ahora estoy en el Ministerio de Seguridad, pero estoy en un curso, no estoy trabajando 
todavía. Me están pagando pero yo estoy haciendo un curso. En el curso veo leyes, me capacitan, redacción. Sería una capacitación pero me pagan. Y voy seis horas pero tengo que cumplir, tengo que estar a horario y el curso es como una especie de facultad: vos cursas y a fin de año tenés que rendir los finales. Ahora tenemos parciales. Hay un poco de derecho penal, hay un poco de todo. Y yo voy haciendo a la par un poquito de la facultad. Son dos años [del curso] y yo voy terminando primero. Una vez que terminás, ya te acomodás mejor. Podés hacer 12 horas por 24 horas así tenés un día libre, podés tener otro trabajo. Si te recibís te pagan el título: es una buena oportunidad. Mal o bien, el trabajo del Estado tiene eso, a menos que te mandes una macana grande, es difícil que te rajen." (Varón, 21 años, GBA)

En particular, debido a la capacitación al que ingresa este joven, su trabajo se relaciona nuevamente con el estudio, visualizando los estudios como trabajo. Aquí a esto se le suma el sector en el que el joven se inserta -el Estado- que le otorgaría al ex becario, desde su perspectiva, la estabilidad que busca y necesita.

Un último factor que nos interesa resaltar respecto de la relación al abandono del Programa debido a la posibilidad o necesidad de trabajar, es que como aparece en varios de los fragmentos arriba citados, personas que ya no forman parte del Programa manifiestan ciertos niveles de presión y control sobre ellos y su trayectoria universitaria que, a diferencia de lo que sucede con la mayoría de las personas becadas activas que perciben como una instancia de control o "presión positiva", entre los estudiantes que ya no integran el Programa es identificada como una "presión negativa". En este sentido, trabajar aparece como aquello que les permite seguir estudiando a sus propios tiempos y según sus posibilidades y exigencias, sin rendirle cuentas a nadie excepto a ellos mismos. De manera que en varios de los relatos, la posibilidad que permite el trabajo, además de la independencia en términos económicos o la necesidad de trabajar, es la posibilidad de emanciparse de estructuras colectivas que tal vez no se ajustan a su realidad cotidiana. Como lo afirma un ex becario:

"No, yo básicamente me sentía un poco inseguro, porque podía haber seguido estudiando, ellos me lo propusieron pero yo me sentía inseguro por no poder dar todas las materias que ellos me pedían. Y ese fue el punto por el cual no seguí en el Programa." (Varón, 20 años, GBA) 
Antes de pasar al próximo repertorio, en función de los fragmentos de las entrevistas y las dimensiones relevadas, resulta pertinente reflexionar respecto de la relación entre trabajo y educación que aparece en los discursos de los jóvenes. Por un lado, pareciera que existe en los casos de los ex becarios analizados cierta tensión entre el trabajo y la educación que se resuelve a favor del primero, al menos momentáneamente, vale decir que si bien estos jóvenes no dejan sus estudios, priorizan el trabajo, como lo adelantábamos en el título de este apartado. Esta tensión podría también expresarse en términos de beneficios inmediatos versus los diferidos que suelen expresarse a través de los estudios: mientras que mediante el trabajo los jóvenes acceden a derechos y beneficios concretos que se traducen en su realidad cotidiana e inmediata, la propuesta de continuar los estudios -siguiendo las trayectorias ideales pautadas de acuerdo a las propuestas del Programa y las universidadesaparece como restrictiva de los mismos. Sin embargo, también es importante resaltar que los jóvenes en cuestión no abandonan sus carreras, sino que su trayectoria combina pausas o abandonos momentáneos con ritmos de cursada diferentes, sólo para listar algunos de sus modos de transitar los estudios superiores. Mediante estos recorridos alternativos en primer lugar, muestran formas alternativas o diferenciadas de relacionarse con la universidad.

Luego, ligado a esto, vale la pena la reflexión acerca de los motivos por los que estos jóvenes continúan con sus estudios. Sobre esto, podemos afirmar que si bien el trabajo ocupa un lugar definitorio en la construcción identitaria, en Argentina, el sistema educativo es el principal dispositivo a partir del cual se procesa la igualdad (Dussel, 2005). Dicho de otra manera, la sociedad argentina ubicó y ubica al sistema educativo como la principal instancia a partir de la cual la afirmación de igualdad y dónde movilidad ascendente tiene a procesarse, aun cuando la realidad no se muestre siempre en sincronía con tal afirmación.

\subsubsection{La distancia de la realidad del becario y el sentimiento de incomprensión}

Un argumento que aparece en algunas entrevistas, aunque de mucha menor intensidad y frecuencia, refiere a ciertos niveles de incomprensión por parte de la OSC a cargo de su beca respecto de las necesidades y realidad de las personas becadas. Un ex becario relata una situación:

“Una vez también, yo me quería comprar una bicicleta porque la mía ya estaba mal y me pasé [del monto que la OSC reconocía para los gastos]. Pero qué pasa, como es acumulativo... vos te quedabas con la plata del mes pasado y podías usarlo si es que te pasabas, entonces lo hacía. Y bueno sí me pasé, y bueno hubo una llamadita de 
atención por ahí. Porque yo no había consultado la compra de esa bicicleta. Entonces así, como que yo no vi muy importante tener que pedir permiso para comprarme una bici, pero bueno... si ellos son estrictos voy a cumplir las reglas, ¿qué voy a hacer? $Y$ bueno así fui desenvolviéndome." (Varón, 20 años, GBA)

En este mismo joven, la sensación de incomprensión se redobla con los controles respecto de qué erogaciones pueden estar comprendidas dentro del gasto del estipendio y cuáles no, algo que además no aparece estandarizado en todas las OSC. Más allá de esto, aquí aparece un conflicto respecto de qué cubría la beca y qué no, y la forma en que se le depositaba el estipendio:

"Lo que me incomodaba era la forma de pago. Porque vos primero comprabas, después rendías y después te daban la plata. Y todo lo del primer cuatrimestre me lo banco mi viejo... siempre tenía que tener plata yo para financiarme, y como que ya en la casa estaba todo medio medio, y mi viejo gastó mil pesos y después me dieron \$ 750 entonces quedaba esa plata y yo que iba y se la pedía a un vecino para devolverle esa plata... tenía que devolver y ¿para ese mes que me quedaba? Entonces tenía que de vuelta pedir plata... económicamente era ese el problema. Si me hubieran depositado directo la plata que mi viejo usaba conmigo la podría haber usado para otras cosas en casa." (Varón, 20 años, GBA)

En el marco de esta situación, agregado a que estaba trabajando, el entonces becario comienza a cuestionarse su continuidad en el Programa:

Investigadora: Cuando pensaste en dejar, ¿qué hiciste?

Becario: Fui para allá, lo hablé con quién era mi tutora.

I: ¿Vos no estás en condiciones de seguir?

B: Bueno está bien. Y bueno ahí fue cuando me dijo: ¿¿No pensas hacer nada para lograr mantener la beca?' Y le dije que no, porque no estaba dispuesto a hacer ningún esfuerzo... no podía dejar de trabajar. Porque no llegaba con las materias también... pero ya a lo último también... la conversación esa fue muy tensa porque también en un momento me dijo algo así como ‘¿Quién te pensás que sos?’ Porque me decía que cuando ella me hablaba la miraba con cara de que no me importa nada. Pero yo no hice la mirada así... porque cuando entra eso en una conversación que supuestamente debería ser serena iya está! Y dije no: - No te voy a discutir." 
Los fragmentos incluidos anteriormente denotan cierta imposibilidad de la OSC por comprender las necesidades del becario. Es decir, la OSC ponderó como de mayor importancia algunos aspectos administrativos y de control, por sobre las necesidades reales del estudiante y su realidad familiar.

En otro trabajo (Mayer y Cerezo, 2014), hemos analizado los modos en que se vinculan las personas becadas, el Programa y los agentes institucionales de las OSC. En particular, elaboramos una tipología de jóvenes becados, en función a las expectativas de las OSC para desarrollar el Programa. Allí describimos tres tipos de personas becadas: en primer lugar, el becario "honorífico", de alta afiliación e identificación con el Programa, caracterizado por una alta participación en el mismo al tiempo que una creencia en sus "dones" y capacidades individuales que le valían si inclusión en el Programa. Luego, en el otro extremo, el becario "pragmático", caracterizado por una identificación baja con el Programa, que muchas veces incluía no saber de dónde provenía su financiamiento y que participaba sólo de las propuestas institucionales para mantener su beca. En el medio de estos tipos, encontrábamos el becario "intermitente" cuya participación en la estrategia podía moverse de manera pendular entre ambos extremos, según situaciones específicas. Así mismo, sosteníamos que esta tipología de becarios era estructurada por la mutua determinación y encuentro de la gramática institucional de cada OSC y la reciprocidad de sentido (Noel, 2007) por parte de las personas becadas. Dicho de otra manera, encontrábamos un sistema de expectativas recíproco entre las expectativas de las OSC respecto de cómo debían comportarse los jóvenes becados, al tiempo que éstos encontraban plataformas más o menos acordes a lo que significaba para ellos ser becario.

En la medida en que la selección para incluir a un aspirante al Programa está en manos de cada OSC, basándose en los criterios generales arriba establecidos de la entidad financiera, cada institución elegía a becarias y becarios según su perfil "ideal” y orientado mayoritariamente, a alguno de estos tipos ideales descritos dependiendo de la organización. Lo cierto es que más allá de ciertas excepciones en sus procesos de selección, las OSC tendían a homogeneizar a las personas becadas en función a diferentes criterios, que derivan en una composición mayoritaria -aunque no total- a favor de uno de los tres tipos mencionados, y en algunos casos, se avizoraba cierta rigidez frente a personas becadas que excedían tales definiciones o bien que no se ajustaban a las mismas, como es el caso de los "ex becarios". Entre ellos parecería ser que cuándo un estudiante no puede adaptarse a los requerimientos de la OSC, ante esta severidad institucional, la relación se tensa hasta 
inclusive, romperse, mostrando un sistema de expectativas no recíprocas respecto de cuáles son los requerimientos, determinaciones y delimitaciones de cada rol.

\section{Conclusiones}

A partir del recorrido presentado respecto de los motivos por los cuales algunos integrantes del Programa de apuntalamiento a las trayectorias universitarias dejaron de pertenecer al mismo, es decir pasaron de ser personas becadas a "ex becadas", pudimos agrupar esas motivaciones en dos grupos.

El primero refiere a un apartamiento del Programa por decisión institucional, es decir que las OSC que gestionan las becas decidieron poner fin a la participación de ese integrante. Dentro de este tipo de apartamiento encontramos a aquellas personas becadas que fueron "invitadas" a abandonar el Programa porque no alcanzaron a aprobar las 4 materias anuales establecidas por el Programa para garantizar su permanencia dentro de la estrategia. Esto se vincula a la distancia existente entre las demandas de las universidades prácticas y la cultura universitaria - y la realidad individual y familiar de estos jóvenes, así como también a la falta estrategias de las instituciones, ya sea educativas como las mismas OSC, para fortalecer su trayectoria, o bien una combinación de ambos aspectos. En este punto planteábamos la convivencia existente entre OSC que parecieran estar más pegadas a la normativa del Programa para su implementación y aquellas que intentan flexibilizar ciertas reglas ante casos especiales. Por otra parte reflexionábamos que muchas veces la resolución de estos casos tiene que ver con el esfuerzo (o no) de la OSC y que esta laxitud puede generar situaciones de malestar e inequidad para con el resto de los participantes que sí lograron cumplir con los requisitos. Esta disyuntiva entre generar mayores niveles de inclusión a través de flexibilizar las normas o mayor adhesión a las mismas, lo que desemboca en problemas en la permanencia, es un debate presente en las políticas educativas argentinas con la vuelta de la democracia. Como sostiene Kessler (2015), la agenda postdictadura estuvo signada por el debate entre inclusión y calidad educativa, estando hasta principios del nuevo milenio, el eje en la inclusión. Si bien muchos agentes educativos se refieren en la actualidad a la "inclusión con calidad" (Misirlis, 2009), el debate respecto de generar más desigualdades dentro de plataformas incluyentes o sistemas más homogéneos que dejan las desigualdades por fuera de los programas específicos no parece estar saldado y es lo que las estrategias diferenciales de las OSC estarían reflejando. Dicho en palabras de Rawls (1993), en sociedades que valoran la igualdad de las personas, 
adquiere importancia -o no- la construcción de desigualdades justas. Aquí el mérito ocupa un lugar importante, en la medida que justifica las acciones individuales diferentes sobre marcos igualitarios e igualadores. Inclusive con todos los cuidados del caso, Rawls (1993) sostiene que el mérito es el mejor mecanismo de construir desigualdades justas. Esta visión estaría asociada a las OSC con perfiles más normativos, que tomarían el mérito como forma productiva de combinar iguales condiciones con los resultados esperados como principio de justicia y luego, de permanencia.

La elección de jóvenes de otras carreras no financiables por el Programa corresponde a otro de los desencadenantes de una desvinculación. Cabe referir que esta situación no necesariamente implica una ruptura del vínculo con la OSC, ya que muchas veces puede relocalizar a ex becarios en otras estrategias que acepten la nueva elección.

Desde nuestra perspectiva, las acciones que desarrollan las OSC para flexibilizar o reubicar a los participantes del Programa, pueden leerse también en clave a discusiones contemporáneas respecto de la exclusión y vulnerabilidad social (Castel, 1997, Sen 2000) donde si bien se reconoce la importancia de las condiciones materiales, también se focaliza en la importancia de la red de relaciones o capital social para que los individuos puedan transitar hacia condiciones más favorables. Que haya habido casos de esfuerzos de gestión por parte de algunas OSC para que, pese a los cambios de carrera o inclusive, pese a no cumplir con los requerimientos, los participantes puedan permanecer en el Programa, indicaría también el esfuerzo por sostener vinculaciones específicas que engrosan los tejidos sociales de los y las jóvenes. Incluso, esto se ve en intentos de OSC para que los participantes permanezcan aún sin la prestación económica. Esto se inscribe dentro de debates todavía no saldados dentro de la investigación educativa, respecto de la incidencia de las tutorías en el marco de programas de inclusión universitaria (García de Fanelli, 2014).

El segundo grupo de motivaciones para apartarse del Programa engloba a aquellas decisiones de las propias personas becadas. El primero de los repertorios enunciados por los ex becarios se vincula al "altruismo" en tanto deciden dejar el Programa porque otro joven podría utilizar mejor los recursos, entienden que la prestación económica dejó de ser necesaria para ellos. Las narrativas incluidas en este grupo, responden también a cierta lógica de filantropía social que existe en el marco del Programa.

Otro de los argumentos por los cuales refieren haber dejado el Programa se vincula a las dificultades de alcanzar los requisitos académicos de permanencia. De modo que optan por salirse del Programa pero continuar sus estudios universitarios, aunque a su propio ritmo. 
Al respecto advertíamos que esta modalidad de apartamiento se vincula, no solo a las falencias en la formación de niveles de enseñanza anteriores, sino también a las imposibilidades de las universidades para garantizar el aprendizaje y permanencia de este tipo de los estudiantes que se alejan del "ideal".

La imposibilidad de tener un trabajo, según lo exige el Programa, aparece como una restricción fundamental para la continuidad dentro del mismo, sobre todo cuando el estipendio mensual no es suficiente para adquirir los recursos necesarios para el desarrollo de la cotidianeidad de la trayectoria universitaria. El trabajo de García de Fanelli (2014) muestra coincidencias en este punto.

De modo que algunos estudiantes optan por priorizar el trabajo y continuar los estudios pero en los tiempos que les permitan combinar trabajo y estudio. Priorizar el trabajo y continuar los estudios es posible por ciertas características de las universidades nacionales (gratuidad, posibilidades de diversos horarios de cursada, sensación de que estarán "siempre alli" y por lo tanto sus trayectorias universitarias pueden combinar períodos de cursada y trabajo con otros de sólo trabajo). Trabajar les permite, no solo cubrir los gastos corrientes que implica ser estudiante -aspecto que cubría la beca- sino también contribuir con la economía de sus hogares y/o realizar otro tipo de gastos, así como también gozar de aquellos derechos que implica contar con un trabajo formal. En este sentido, trabajar aparece como aquello que les permite seguir estudiando a sus propios tiempos y según sus posibilidades y exigencias, sin rendirle cuentas a nadie excepto a ellos mismos.

Desde nuestra perspectiva, el abandono al no poder alcanzar los requisitos y expectativas del Programa como por la insuficiencia del monto del estipendio frente a posibilidades laborales -y todos sus beneficios materiales y simbólicos- también ilumina sobre otra cuestión: atender en qué medida las acciones complementarias aquí desplegadas, que son similares a las propuestas por programas estatales, son suficientes para paliar los déficits de conocimiento -acarreados principalmente desde el nivel medio- y del capital cultural necesario para transitar exitosamente las instituciones de educación superior, por las constricciones propias del dispositivo.

Por último, con mucha menor intensidad y frecuencia, refiere a ciertos niveles de incomprensión por parte de la OSC a cargo de su beca respecto de las necesidades y realidad de las personas becadas y una sensación de incomprensión. A partir de un análisis de los modos en que se vinculan los becarios, el Programa y los agentes institucionales de las OSC observamos que existe una tipología de personas becadas estructurada por la 
mutua determinación y encuentro de la gramática institucional de cada OSC y la reciprocidad de sentido. Parecería ser que cuándo un estudiante no puede adaptarse a los requerimientos de la OSC, ante esta severidad institucional, la relación se tensa hasta inclusive, romperse, mostrando un sistema de expectativas no recíprocas respecto de cuáles son los requerimientos, determinaciones y delimitaciones de cada rol.

Aquí podemos referirnos a un problema generalizado de los sistemas de tutorías, tanto en este Programa como en otros, donde inclusive estudiantes avanzados ejercen ese rol (García de Fanelli, 2014). En muchos casos, ante la ausencia de conocimientos específicos, quienes desarrollan estas prácticas lo hacen desde su propio sentido común o desde definiciones institucionales (IIPE-UNESCO y PNUD, 2009), es decir, motorizando sus buenas intenciones al tiempo que prejuicios y su propia experiencia como estudiantes. Más allá de su buena voluntad, esto puede derivar en graves falencias respecto a su desenvolvimiento. El despliegue de las tutorías al igual que las becas, resulta consistente con la delimitación del problema -abandono y/o dificultad de la permanencia- sin muchas veces dotar de sentidos específicos a los agentes que desarrollan estas prácticas y si bien pueden compensar falencias relacionados a la integración académica, difícilmente puedan paliar las relacionadas a la integración social. Más aún, la distancia social puede hacerse presente y agravar la posibilidad de desarrollar vínculos (Tinto, 1975).

\section{Referencias}

Argentina, Ministerio de Educación, Secretaría de Políticas Universitarias. (2013). Anuario de la Secretaría de Políticas Universitarias. CABA, Argentina: SPU.

Beck, Ulrich. (2001). Repensar lo político. Madrid, España: Alianza.

Beech, Jason e Ignacio Barrenechea. (2011). Pro- market educational governance: is Argentina a black swan? Critical Studies in Education, 52(3), 279-293.

Bourdieu, Pierre y Passeron, Jean-Claude. (2009). Los herederos. Los estudiantes y la cultura (2 ${ }^{\mathrm{a}}$. ed.). Buenos Aires: Siglo XXI Editores.

Bourdieu, Pierre. (1999). La miseria del mundo. Buenos Aires: Akal ediciones.

Buchbinder, Pablo. (2005). Historia de las universidades argentinas. Buenos Aires: Sudamericana.

Carli, Sandra. (2012). El estudiante universitario: Hacia una historia del presente de la educación pública. Buenos Aires: Siglo XXI Editores.

Castel, Robert. (1997). La metamorfosis de la cuestión social. Buenos Aires: FCE. 
Coulon, Alain. (2005). Le métier d'étudiant: l'entrée dans la vie universitaire, París: Economica-Anthropos.

Duschatzky, Silvia. (1999). La escuela como frontera: reflexiones sobre la experiencia escolar de jóvenes de sectores populares. Buenos Aires: Paidós.

Dussel, Inés. (2005). Desigualdades sociales y desigualdades escolares en la Argentina de hoy. Algunas reflexiones y propuestas. En Juan Carlos Tedesco (comp), ¿Cómo superar la fragmentación del sistema educativo argentino? (pp. 85 - 116). Buenos Aires: IIPE- UNESCO Ediciones.

Ezcurra, Ana María. (2009). Educación Universitaria: una inclusión excluyente. Buenos Aires: Fundación IDEAS.

Ezcurra, Ana María. (2011). Igualdad en educación Superior. Un desafío Mundial (Serie Universidad). Los Polvorines, Buenos Aires: UNGS, IEC-CONADU.

Fernández Prieto, Anabel y Cerezo, Leticia. (2010) Manual de planificación, monitoreo y evaluación. Buenos Aires: Programa Remediar + Redes del Ministerio de Salud de la Nación Argentina.

García de Fanelli, Ana. (2005). Acceso, abandono y graduación en la educación superior argentina. Sistema de información de tendencias educativas en América Latina. Buenos Aires: IIPE UNESCO.

García de Fanelli, Ana y Jacinto, Claudia. (2010). Equidad y educación superior en América Latina: el papel de las carreras terciarias y universitarias. Revista lberoamericana de educación superior, 1(1), 58-75.

García de Fanelli, Ana. (2014). Inclusión Social en la Educación superior argentina: Indicadores y políticas en torno al acceso ya la graduación. Páginas de Educación, 7(2), 124-151.

García de Fanelli, Ana y Adrogué, Cecilia. (2015). Abandono de los estudios universitarios: dimensión, factores asociados y desafíos para la política pública. Revista Fuentes, (16), 85-106. Doi: http://dx.doi.org/10.12795/revistafuentes.2015.i16.04

Gazzola, Ana Lucía y Didriksson, Axel. (2008). Tendencias de la Educación Superior en América Latina y el Caribe. Caracas: IESALC-UNESCO. Recuperado de http://flacso.redelivre.org.br/files/2012/08/753.pdf

Giddens, Anthony. (1998). La Constitución de la sociedad. Bases para la teoría de la estructuración. Buenos Aires: Amorrortu.

Guadagni, Alieto y Boero, Francisco. (2015). La educación argentina en el siglo XXI. Los desafíos que enfrentamos. Buenos Aires: El Ateneo. 
Herrero, Alejandro. (2010). Una aproximación a la historia de la educación argentina entre 1862 y 1930, en los niveles primario y secundario. En Daniel Toribio (comp.), La universidad en la Argentina: miradas sobre su evolución y perspectivas (pp. 31-91). Lanús, Argentina: Universidad Nacional de Lanús, Departamento de Planificación y Política Pública.

IIPE-UNESCO y PNUD. (2009). Abandono Escolar y políticas de inclusión en la educación secundaria. Buenos Aires: IIPE UNESCO Ediciones

Jacinto, Claudia y Terigi, Flavia. (2007). ¿Qué hacer ante las desigualdades en la educación secundaria? Aportes de la experiencia latinoamericana. Buenos Aires: Santillana / IIPEUNESCO.

Jares, Xesús. (1991). El lugar del conflicto en la organización escolar. Revista de educación, (302), 113-128.

Kessler, Gabriel. (2015). Controversias sobre la desigualdad: Argentina, 2003-2013. Buenos Aires: Fondo de Cultura Económica.

Mayer Liliana y Cerezo, Leticia. (2014). Evaluación de Impacto del Programa Potenciamos Tu Talento. Buenos Aires: Asociación Civil Grupo Puentes.

Mayer, Liliana y Cerezo, Leticia. (2016). Tutorías y estipendio mensual: contribuciones a la trayectoria universitaria de jóvenes en situación de vulnerabilidad. Revista Latinoamericana de Ciencias Sociales, Niñez y Juventud, 14(2), 1421-1433.

Mayer, Liliana. (2012). La escuela y la conflictividad cotidiana. Un análisis de las estrategias para su prevención y/o minimización (Tesis para optar por el grado de Doctorado en Ciencias Sociales). Universidad de Buenos Aires, Buenos Aires, Argentina.

Misirlis, Graciela. (2009). Deudas y desafíos en la educación de jóvenes y adultos: una mirada desde un enfoque político-didáctico. Recuperado de http://www.oei.es/historico/noticias/spip.php?article4548

Montes, Nancy y Sendón, María Alejandra. (2006). Trayectorias educativas de estudiantes de nivel medio. Argentina a comienzos del Siglo XIX. Revista Mexicana de Investigación Educativa, 11(29), 381-402.

Montes, Nancy y Sendón, María Alejandra. (2010). Trayectorias educativas de adolescentes en una Argentina fragmentada. Sociología de la educación, (14). Recuperado de http://www.emdialogo.uff.br/sites/default/files/GT14-1926--Int1.pdf

Nirenberg, Olga. (2010). Enfoques para la evaluación de políticas públicas. En Paula Amaya (comp.), El Estado y las Políticas Públicas en América Latina (pp. 227-268). La Plata: Editorial UNLP.

Noel, Gabriel. (2007). Los conflictos entre agentes y destinatarios del sistema escolar en escuelas públicas de barrios populares urbanos (Tesis para optar por el grado de Doctorado en Ciencias Sociales). Universidad Nacional de General Sarmiento, Buenos Aires, Argentina. 
Pochulu, Marcel David y Williams, Gareth. (2004). La educación superior argentina hoy, entre instituciones que se transforman y relaciones que se modifican. Revista Iberoamericana de Educación. Recuperado de https://www.google.com/url?sa=t\&rct=j\&q=\&esrc=s\&source=web\&cd=1\&ved=2ahUKEw iC njxcHaAhWPv1MKHfMCLIQFjAAegQIABAq\&url=https\%3A\%2F\%2Frieoei.org\%2Fhistorico\%2Fdeloslectores\% 2F796Pochulu.PDF\&usg=AOvVaw2bx4Omn3LRicnS5isJnNj8

Rawls, John. (1993). Teoría de la Justicia. Buenos Aires: Fondo de Cultura Económica.

Saltalamacchia, Homero. (1992). La Historia de Vida: Reflexiones a partir de una experiencia de investigación (Colección Investigaciones). Puerto Rico: Ediciones SIJUP.

Sen, Amartya. (2009). The idea of justice. Cambridge, Massachusetts: The Belknap Press of Harvard University Press.

Sverdlick, Ingrid. (2013). Universidad, desigualdad y justicia social. Revista de Educação Pública, 22(48), 63-79.

Tenti Fanfani, Emilio. (2007). La escuela y la cuestión social. Buenos Aires: Siglo XXI.

Terigi, Flavia. (mayo, 2007). Los desafíos que plantean las trayectorias escolares. Paper presentado en el III Foro Latinoamericano de educación. Jóvenes y docentes. La escuela secundaria en el mundo de hoy. Buenos Aires: Fundación Santillana.

Tinto, Vicent. (1975). Dropout from Higher Education: A Theoretical Synthesis of Recent Research. Review of Educational Research,45,(1), 89-125.

Tinto, Vincent. (1989). Definir la deserción: una cuestión de perspectiva. Revista de Educación Superior, 18(71). Recuperado de http://publicaciones.anuies.mx/pdfs/revista/Revista71 S1A3ES.pdf

Tinto, Vincent. (2006). Research and practice of student retention: what is next? Journal of college student retention, 8(1), 1-19.

Tiramonti, Guillermina. (2008). Introducción. En Guillermina Tiramonti y Nancy Montes (Comps.), La escuela media en debate (pp. 9-11) Buenos Aires: Manantial.

Toribio, Daniel. (2010). La expansión de la educación superior en contextos de crisis sociales y políticas. En Daniel Toribio (comp.), La universidad en la Argentina: miradas sobre su evolución y perspectivas. Lanús, Argentina: Universidad Nacional de Lanús, Departamento de Planificación y Política Pública.

Urresti, Marcelo. (1999). Cambio de escenarios sociales, experiencia juvenil urbana y escuela. En Emilio Tenti Fanfani, (comp.), Una escuela para los adolescentes. Materiales para el profesor-tutor (pp. 9-71). Buenos Aires: UNESCO, Ministerio de Educación de la Provincia de Santa Fe y UNICEF, Oficina de Argentina. 